This is a author version of

D. Schlipf, P. Fleming, S. Kapp, A. Scholbrock, F. Haizmann, F. Belen, A. Wright, and P. W. Cheng,

"Direct Speed Control Using LIDAR and Turbine Data,"

published in Proceedings of the American Control Conference, Washington, USA, 2013.

\title{
Direct Speed Control Using LIDAR and Turbine Data
}

\author{
David Schlipf ${ }^{1}$, Paul Fleming ${ }^{2}$, Stefan Kapp ${ }^{1 \ddagger}$, Andrew Scholbrock ${ }^{2}$, Florian Haizmann ${ }^{1}$, \\ Fred Belen $^{3}$, Alan Wright ${ }^{2}$, and Po Wen Cheng ${ }^{1}$
}

\begin{abstract}
LIDAR systems are able to provide preview information of the wind speed in front of wind turbines. One proposed use of this information is to increase the energy capture of the turbine by adjusting the rotor speed directly to maintain operation at the optimal tip-speed ratio, a technique referred to as Direct Speed Control (DSC). Previous work has indicated that for large turbines the marginal benefit of the direct speed controller in terms of increased power does not compensate for the increase of the shaft loads. However, the technique has not yet been adequately tested to make this determination conclusively. Further, it is possible that applying DSC to smaller turbines could be worthwhile because of the higher rotor speed fluctuations and the small rotor inertia. This paper extends the previous work on direct speed controllers. A DSC is developed for a $600 \mathrm{~kW}$ experimental turbine and is evaluated theoretically and in simulation. Because the actual turbine has a mounted LIDAR, data collected from the turbine and LIDAR during operation are used to perform a hybrid simulation. This technique allows a realistic simulation to be performed, which provides good agreement with theoretical predictions.
\end{abstract}

\section{INTRODUCTION}

LIDAR (LIght Detection And Ranging) systems are able to provide information about the wind field inflow, such as wind speed, shears, and wind direction, which can be used to assist wind turbine control. There have been numerous ongoing studies into the means by which LIDAR can improve wind turbine control performance. LIDAR-assisted collective pitch control for load reduction is one option and has actually been demonstrated recently in field testing [1], [2]. Another potential beneficial use of LIDAR is to increase energy yield by LIDAR-assisted yaw control [3], [4].

Another possibility to increase the energy production of wind turbines with LIDAR is by a "direct speed controller" (DSC) as proposed in [4], which minimizes the standard deviation of the tip speed ratio (TSR) and can be implemented as a simple update to commonly used variable speed controllers. By more tightly controlling the TSR, the energy production of the turbine is expected to rise. However, an important disadvantage to using DSC is the higher loads on the shaft due to the necessarily higher fluctuations in the generator torque.

In this paper, data collected from ongoing field tests of LIDAR-enhanced collective pitch control on an experimental

\footnotetext{
${ }^{1}$ Stuttgart Wind Energy Research (SWE), Universität Stuttgart, Germany, David.Schlipf/Florian.Haizmann/PoWen.Cheng at ifb.uni-stuttgart.de, ${ }^{\star}$ now with Robert Bosch GmbH

2 National Renewable Energy Laboratory (NREL), USA Paul.Fleming/Andrew.Scholbrock/Alan.Wright at nrel.gov

${ }^{3}$ Blue Scout Technologies, USA fbelen@bluescout. com
}

wind turbine is used to evaluate the trade-offs involved with the inclusion of DSC. Data collected from the commercial LIDAR device installed on the nacelle of the CART3 (Controls Advanced Research Turbine, 3-Bladed) turbine at the National Renewable Energy Laboratory (NREL) is used together with the rotor effective wind speed extracted from the CART3 sensor data to simulate and optimize the DSC for this case. By using data from a physical turbine and LIDAR operating in turbulent inflow, the paper provides a realistic examination into the benefits and detriments of using a DSC.

This paper is organized as follows. Section II summarizes the experimental environment. In Section III, the controller design is outlined. The correlation of the LIDAR and the turbine is described in Section IV. Section V presents the simulation with real data, and conclusions and future work are discussed in Section VI.

\section{TEST ENVIRONMENT}

In this section, the test site, turbine, and LIDAR system for the field testing are described.

\section{A. The Test Site}

The field testing takes place at the National Wind Technology Center (NWTC) in Boulder, Colorado, which is part of NREL. Due to its location directly east of the Rocky Mountains Front Range, the NWTC offers good conditions during the wind season in winter to perform any kind of field test. Since the wind conditions are rather gusty and extreme, tests under extreme conditions can especially be performed there.

\section{B. The Test Turbine}

Among several multi-megawatt turbines, the NWTC also owns two mid-sized turbines (CART2 and CART3), which are dedicated to the testing of new and advanced control algorithms of wind turbines. The CART3 (Figure 1) is a $600 \mathrm{~kW}$ variable-speed pitch-controlled turbine that was modified with a three-bladed rotor and currently operates at $550 \mathrm{~kW}$ due to resonance issues [5]. The CART3 is instrumented with strain gauges, accelerometers, and a dedicated meteorological tower, installed $80 \mathrm{~m}$ in front of the turbine's mean wind direction $\left(292^{\circ}\right)$. The turbine has a rotor diameter of $D=40 \mathrm{~m}$, a hub height of $36.6 \mathrm{~m}$, and runs at a rated rotor speed of $37 \mathrm{rpm}$. The control system offers an easy way of implementing new controller code as a DLL, which is then loaded by the $400 \mathrm{~Hz}$ real-time LabVIEW framework control system. For this work, the DLL was created as an export from MATLAB/Simulink code. 


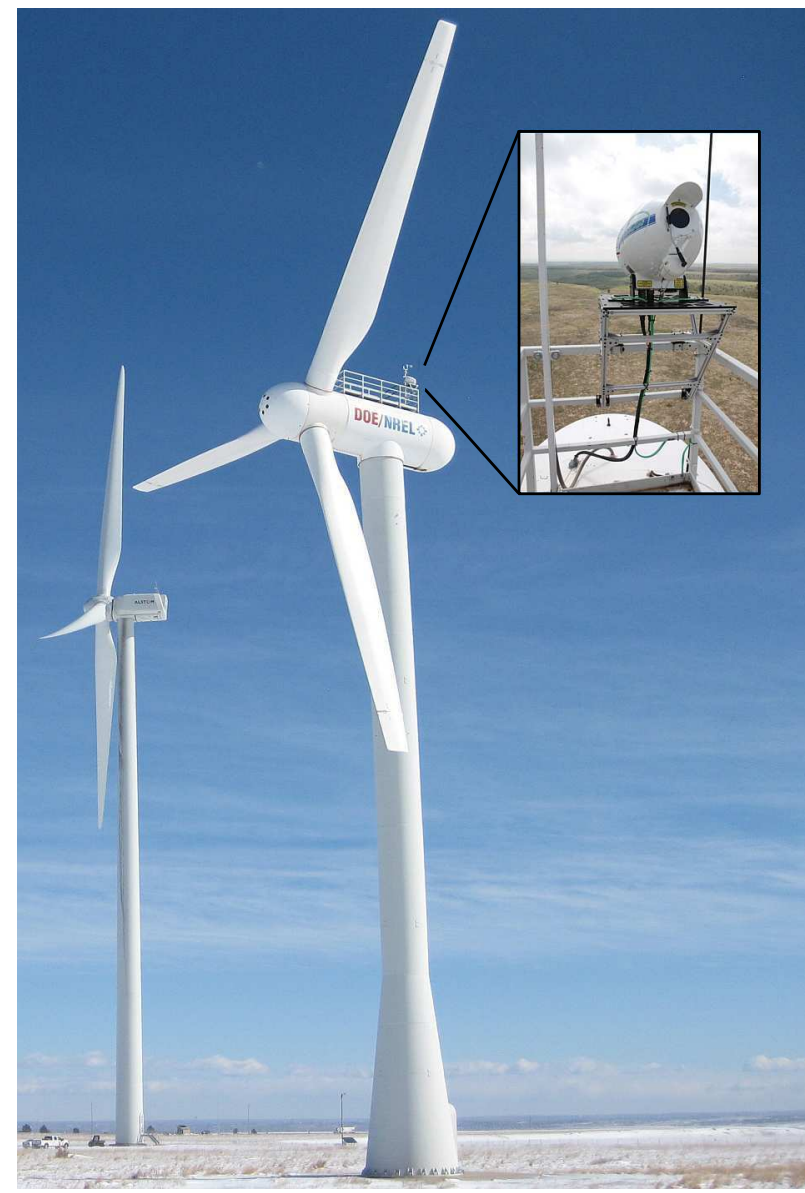

Fig. 1. The OCS installed on the CART3 at the NWTC.

\section{The LIDAR System}

The LIDAR used in these tests to measure the wind in front of the turbine is a commercial, pulsed system from Blue Scout Technologies. It simultaneously measures the Doppler shift of the light backscattered on aerosols at three range gates along the three fixed laser beams. It uses the lineof-sight wind speed to internally derive the horizontal and vertical wind speed and wind direction. For this campaign, all internal processing, such as filtering, has been reduced to the minimum to be able to use it for real-time feedforward and direct speed control. From these multiple inputs, a resulting rotor effective speed is determined by assuming that only the longitudinal wind component is responsible for power generation. Only the first range gate at $x_{1}=40 \mathrm{~m}$ is used in these tests due to interference with the met mast and guy wires of the other range gates.

\section{Controller Design}

The main purpose of variable speed control for wind turbines below rated wind speed is to maximize the electrical power extraction [6]. Therefore, the turbine has to operate with the rotor blades held at the optimal angle of attack. This blade inflow angle is represented by $\lambda$, the ratio of the blade

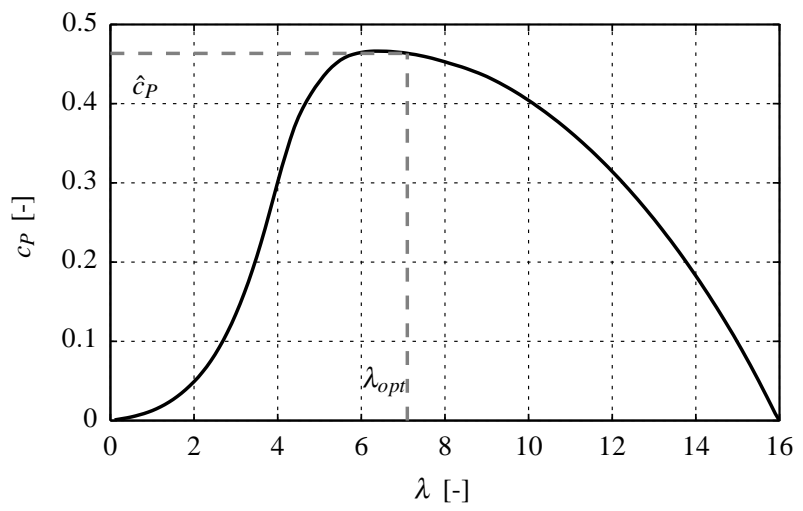

Fig. 2. Power coefficient $c_{P}$ over tip speed ratio $\lambda$ of the CART3.

tip speed and the undisturbed rotor effective wind $v_{0}$ :

$$
\lambda=\frac{\Omega R}{v_{0}},
$$

where $R$ is the rotor radius and $\Omega$ the rotor speed. The optimal tip speed ratio $\lambda_{\text {opt }}$ can be found at the peak $\hat{c}_{P}$ of the power coefficient, which depends below rated wind speed only on $\lambda$ (see Figure 2 for the CART3). The aerodynamic optimum can be achieved by tracking $\lambda_{\text {opt }}$ by adjusting the generator torque $M_{g}$. Typically the torque is set such that TSR is maintained optimal in the static case. This section extends the controller presented in [4] and depicts how tracking $\lambda_{\text {opt }}$ can be done dynamically by using the knowledge of the incoming wind.

\section{A. Indirect Speed Control}

Nonlinear state feedback controllers are commonly used in wind energy to control $\lambda$ indirectly, measuring the generator speed $\Omega_{g}$.

For derivations of state feedback control laws, the following nonlinear reduced model of a turbine for below rated wind speed is chosen based on [6]:

$$
\begin{aligned}
J \dot{\Omega} & =M_{a}-M_{g} / n_{g b} \\
M_{a}\left(\Omega, v_{0}\right) & =\frac{1}{2} \rho \pi R^{3} \frac{c_{P}(\lambda)}{\lambda} v_{0}^{2},
\end{aligned}
$$

where $M_{a}$ is the aerodynamic torque, $n_{g b}=\Omega / \Omega_{g}$ is the gear box ratio, $\rho$ is the air density, $R$ is the rotor radius and $J$ is the sum of the moments of inertia about the rotation axis. The generator torque to maintain in steady state the maximum power coefficient $\hat{c}_{P}$ can then be determined by [6]:

$$
M_{g, \mathrm{ISC}}=\underbrace{\frac{1}{2} \rho \pi R^{5} \frac{\hat{c}_{P}}{\lambda_{o p t}^{3}} n_{g b}^{3}}_{k_{\mathrm{ISC}}} \Omega_{g}^{2} .
$$

Equation (3) with constant $k_{\mathrm{ISC}}$ is known as "Region 2" of the indirect speed control (ISC) and is used as a reference in this study.

Figure 3 shows the defined piecewise ISC state feedback law $\Sigma_{\text {ISC }}$ with linear ramps for the transitions from startup ("Region 1") and to full load ("Region 3"). 


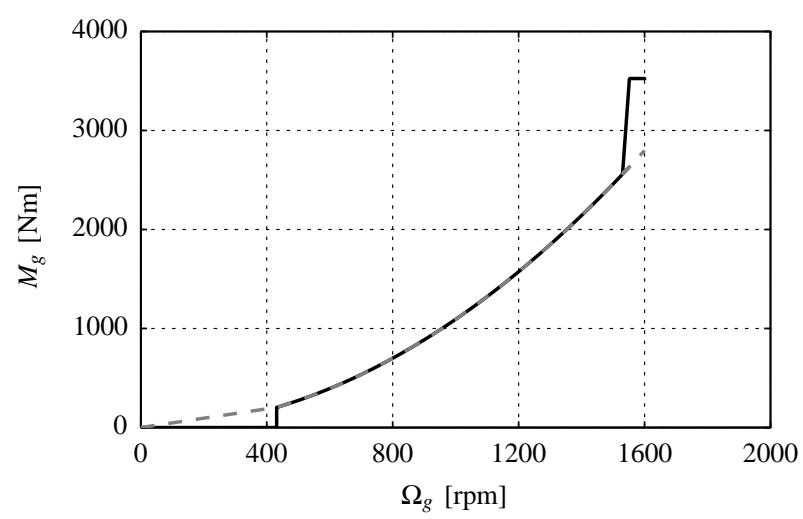

Fig. 3. Optimal relation of rotor speed and generator torque (dashed), state feedback used in control law (solid), Region 2 (overlapping region of two lines).

\section{B. Direct Speed Control}

Using the LIDAR technology, $v_{0}$ and thus $\lambda$ become measurable, and therefore, the proposed controller is considered as direct speed control (DSC), which was presented in [4] and extended in this paper. The basic idea of the proposed DSC is to keep the ISC feedback law (3) and to find a feedforward update to compensate changes in the wind speed similar to the one used for collective pitch control [7]. One advantage of this structure is that the stability behavior of the speed control loop is not modified. Therefore, the rotor speed error $\varepsilon$ is introduced

$$
\varepsilon=\Omega-\Omega_{\text {opt }},
$$

where the optimal rotor speed $\Omega_{o p t}$ is defined as

$$
\Omega_{o p t}=\frac{\lambda_{o p t} v_{0}}{R} .
$$

By setting $\varepsilon=0$, the DSC (see [4] for more details) is

$$
M_{g, \mathrm{DSC}}=M_{g, \mathrm{ISC}} \underbrace{-n_{g b} J \frac{\lambda_{o p t}}{R} \dot{v}_{0}}_{M_{g, \mathrm{FF}}} .
$$

The dynamics of the closed loop is determined by the order of the differential equation of the error. Choosing a first and second order similar to [8]

$$
\begin{aligned}
\dot{\varepsilon}+a_{0} \varepsilon & =0, \\
\ddot{\varepsilon}+a_{0} \dot{\varepsilon}+a_{1} \varepsilon & =0,
\end{aligned}
$$

the direct speed controller can be extended to:

$$
\begin{aligned}
M_{g, \mathrm{PDSC}} & =M_{g, \mathrm{ISC}}+M_{g, \mathrm{FF}}+n_{g b} J a_{0} \varepsilon \\
M_{g, \mathrm{PIDSC}} & =M_{g, \mathrm{ISC}}+M_{g, \mathrm{FF}}+n_{g b} J\left(a_{0} \varepsilon+a_{1} \int \varepsilon d t\right),
\end{aligned}
$$

respectively. Figure 4 shows the closed loop. In addition to (6), the controllers (9) and (10) provide a proportional $\left(a_{0}\right)$ and respectively a proportional and an integral $\left(a_{0}, a_{1}\right)$ feedback factor. The resulting asymptotically stable closed loop dynamics (7) and (8) can be chosen by specifying positive values for the parameters $a_{0}$ and $a_{1}$.

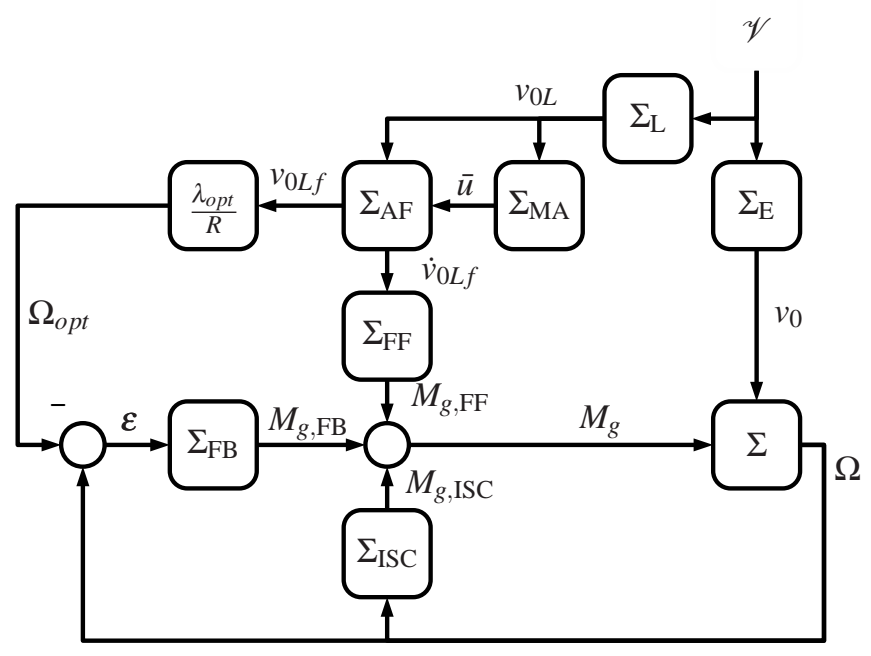

Fig. 4. Scope of the different DSCs.

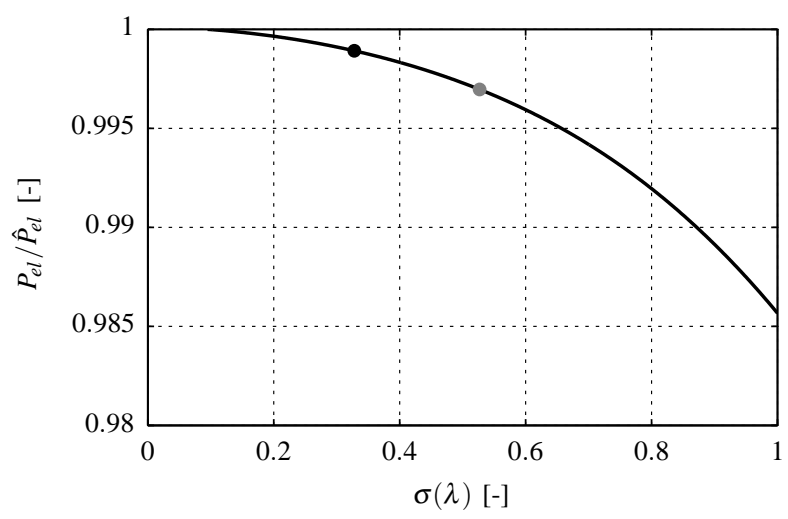

Fig. 5. Relative power extraction by variation in tip speed ratio for the CART3. Dots: Results from Section V, ISC (gray) and DSC (black).

\section{Limits of Direct Speed Control}

The increase in energy production is obtained by improving the accuracy of tracking the optimal tip speed ratio. Therefore, the fluctuation of the tip speed ratio can be used as a measure for the potential of energy optimization. Assuming the distribution of the tip speed ratio $\varphi_{\lambda_{\text {opt }} ; \sigma(\lambda)}$ to be Gaussian with mean $\lambda_{\text {opt }}$ and a standard deviation $\sigma(\lambda)$, then the generated power can be estimated by

$$
P_{e l}(\sigma(\lambda))=\hat{P}_{e l} \int_{-\infty}^{\infty} \varphi_{\lambda_{\text {opt }} ; \sigma(\lambda)} c_{P}(\lambda) d \lambda .
$$

In Figure 5, this potential is quantified for the CART3. High reduction of $\sigma(\lambda)$ by the DSC will therefore only cause a low increase in the power due to the relative flat $c_{P}(\lambda)$ curve. The results in the next sections will confirm these considerations.

\section{Implementation}

The proposed controllers have the advantage that they are simple updates to the ISC and therefore can easily be integrated into the existing control strategy.

It is only necessary to track the optimal tip speed ratio in "Region 2". Therefore, the control update to the feedback 


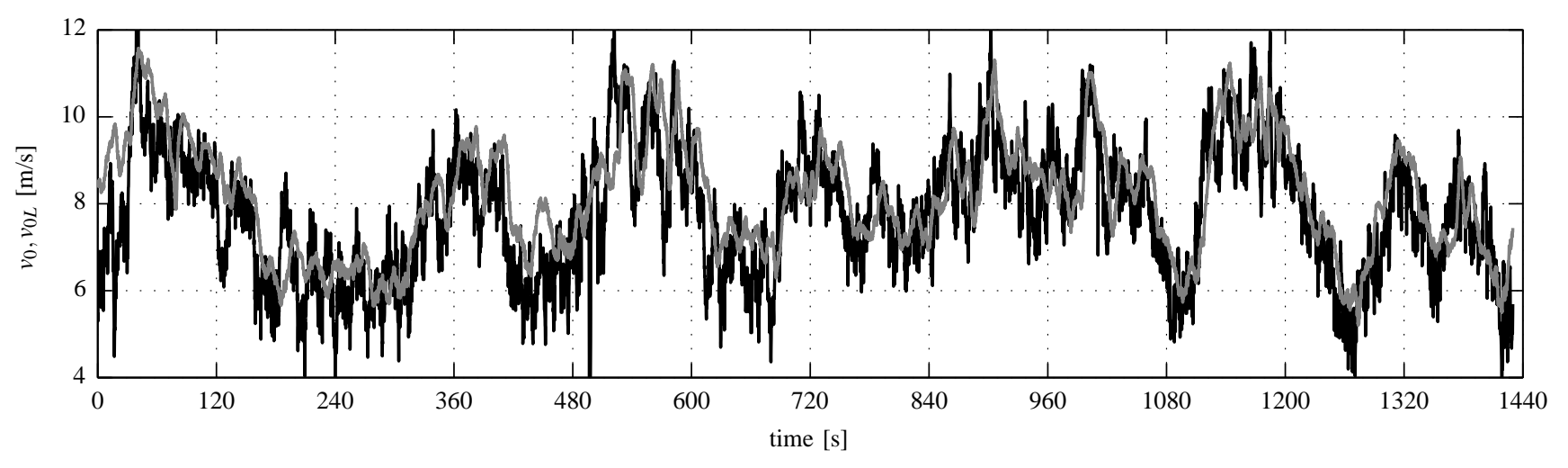

Fig. 6. Comparison of the rotor effective wind speed estimated from LIDAR (black) and turbine data (gray).

controller $\Sigma_{\mathrm{FB}}$ is multiplied with a feedforward window $g_{\mathrm{FF}}$ depending on the rotor effective wind speed. Region 2 is active approximately from $3.3 \mathrm{~m} / \mathrm{s}$ to $11.5 \mathrm{~m} / \mathrm{s}$ for $\rho=1.0 \mathrm{~kg} / \mathrm{m}^{3}$. The feedforward window $g_{\mathrm{FF}}$ is chosen to be 0 for values below $4.5 \mathrm{~m} / \mathrm{s}$ and above $11.5 \mathrm{~m} / \mathrm{s}$ and to be 1 for values between $5 \mathrm{~m} / \mathrm{s}$ and $11 \mathrm{~m} / \mathrm{s}$ with a linear ramp in between, for a smooth transition.

In reality, the disturbance $v_{0}$ to the turbine $\Sigma$ cannot be measured directly and has to be replaced by the LIDAR measurement $v_{0 L}$ (see Figure 4). Both signals are based on the wind field $\mathscr{V}$ in front of the turbine - the LIDAR measurement $\Sigma_{\mathrm{L}}$ yields $v_{0 L}$ and the wind evolution $\Sigma_{\mathrm{E}}$ yields $v_{0}$. An adaptive filter

$$
\Sigma_{\mathrm{AF}}=G_{\text {filter }}(s) e^{-T_{\text {buffer }} s} \approx \Sigma_{\mathrm{E}} \Sigma_{\mathrm{M}}^{-1}
$$

is used to account for the fact that $v_{0 L}$ and $v_{0}$ are only correlated in the low frequencies and to account for the delay due to the propagation of the wind to the turbine. A firstorder Butterworth filter $G_{\text {filter }}$ is fitted to the transfer function $G_{L R}$ estimated from measured data via the auto correlation spectrum of the measured wind speed $S_{L L}$ and the cross correlation spectrum $S_{L R}$ between the measured and the rotor effective wind speed:

$$
\left|G_{\text {filter }}\right| \approx\left|G_{L R}\right|=\left|\frac{S_{L R}}{S_{L L}}\right| .
$$

The filter is parametrized by a static gain $G_{0}$ and a cutoff frequency $f_{\text {cutoff }}=\hat{k} \bar{u} /(2 \pi)$, where $\hat{k}$ is the maximum coherent wavenumber, which has to be determined by a correlation study. The time delay is obtained from the following considerations: With Taylor's hypothesis, the wind needs the time $x_{1} / \bar{u}$ to evolve from the place of measurement to the turbine. Due to the measurement duration $T_{\mathrm{scan}}, v_{0 L}$ is already delayed by $T_{\text {scan }} / 2=1 \mathrm{~s}$ and the filter delay is approximated by $T_{\text {filter}}$. For using the filtered wind in the DSC instead of $v_{0}$, the signal has to be synchronized with $v_{0}$ reaching the rotor plane. Therefore, the necessary time delay is

$$
T_{\text {buffer }}=\frac{x_{1}}{\bar{u}}-\frac{1}{2} T_{\text {scan }}-T_{\text {filter }}-\tau \text {. }
$$

The time $\tau$ can be used to compensate for the slow down of the wind due to the higher pressure in front of the turbine or small errors in the model reduction (see [9]). The mean wind $\bar{u}$ is obtained by a moving average $\Sigma_{\mathrm{MA}}$ over $180 \mathrm{~s}$. Thus, the time delay $T_{\text {buffer }}$ and the filter cutoff frequency $f_{\text {cutoff }}$ is adapted to the mean wind speed and change continuously.

\section{CORRElation Study}

Before applying the DSC to the CART3, a correlation study has been made to determine the maximum coherent wavenumber for the filter design and to adjust $\tau$. Therefore, the measured rotor effective wind speed $v_{0 L}$ from the LIDAR is compared to an estimate from turbine data.

\section{A. The Estimator for the Rotor Effective Wind Speed}

The rotor effective wind speed $v_{0}$ is obtained from simultaneously measured turbine sensor data by an estimator similar to the one presented in [10]. With the reduced system (2) and measured data of $\Omega$, the pitch angle $\theta$ and $M_{L S S}$ (replacing $\left.M_{g} / n_{g b}\right)$, the aerodynamic torque $M_{a}$ can be calculated and reorganized in a cubic equation in $\lambda$ :

$$
\lambda^{3}=\frac{1}{2} \rho \pi R^{5} \frac{c_{P}(\lambda, \theta)}{M_{a}} \Omega^{2} .
$$

Because of the $\lambda$-dependency of $c_{P}$, an explicit expression cannot be found. The equation is solved with a set of $M_{a}, \Omega$ and $\theta$, and a three-dimensional look-up table $v_{0}\left(M_{a}, \Omega, \theta\right)$ is generated, which can then be used to get a time series of $v_{0}$ by a three-dimensional interpolation. The turbine raw data is filtered by notch filters at turbine resonance and disturbance frequencies (such as 3P) before being used.

\section{B. Design of the Adaptive Filter}

For the filter design and the simulations, a 24 min-data set $(21: 44$ - 22:08) from 03/29/2012 is analyzed with a mean wind speed of $8.37 \mathrm{~m} / \mathrm{s}$ and a turbulence intensity of $16.7 \%$ measured by the met mast, fitting to lower turbulence characteristics (Class C) according to current standards. Figure 6 shows the rotor effective wind speed estimated from LIDAR and from turbine data, showing the preview of the LIDAR measurement.

Figure 7 depicts the measured transfer function between the $v_{0}$ and $v_{0 L}$ as well as the chosen filter. The static gain is set to $G_{0}=1$ and the maximum coherent wavenumber, as a compromise, to $\hat{k}=0.0225 \mathrm{rad} / \mathrm{m}$. 


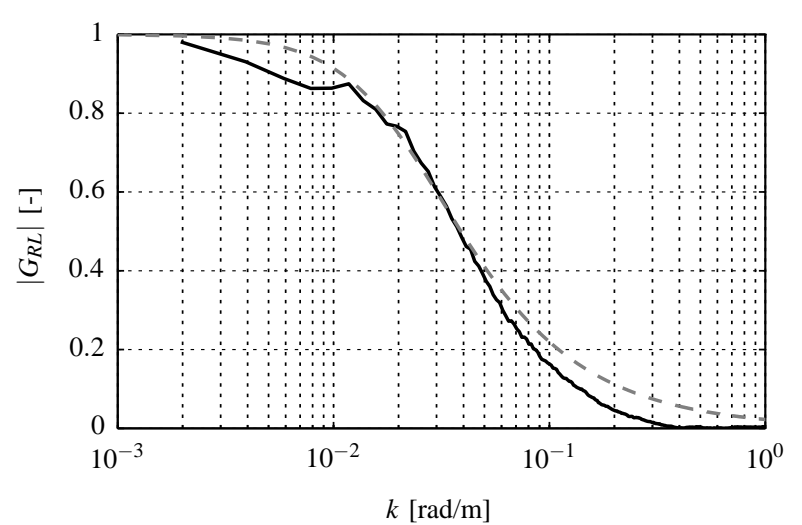

Fig. 7. Transfer function between the turbine and the LIDAR (solid) and the used filter (dashed)

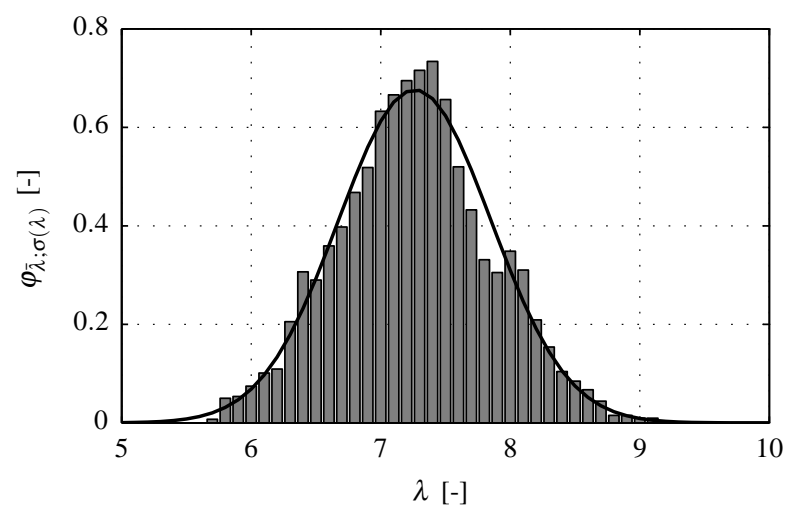

Fig. 8. Histogram and Gaussian distribution of the measured tip speed ratio.

\section{Expected Improvement}

Figure 8 confirms that a Gaussian distribution of the tip speed ratio $\lambda$ is a reasonable assumption. For the data set, the measured mean value is $\bar{\lambda}=7.26$ and the standard deviation $\sigma(\lambda)=0.589$. Neglecting the deviation from $\lambda_{\text {opt }}=7.1$, the applied ISC is already at $99.6 \%$ of the optimal value, according to the theory of Subsection III-C.

\section{HYBRid Simulations}

The LIDAR raw data and the estimated $v_{0}$ are used for simulations to test the DSC. The simulations are done with an aeroelastic model of the CART3 implemented in FAST [11], disturbed by a hub height wind field of $v_{0}$. FAST can be simulated in the MATLAB/Simulink environment, using the same controller that is exported to a DLL for field testing.

This combination of experiment and simulations we call "hybrid simulations". In this case the benefits over conventional simulations with LIDAR simulation and wind evolution models [12] are that effects such as measurement errors and delays, real wind evolution, and site specific problems can be included into the simulations. If used along with the ISC controller, the simulated turbine's reaction will be close to the measured turbine data due to the fact that the used estimation of the rotor effective wind speed $v_{0}$ is an inverse process to the simulation. If used along with the DSC controller, it can be estimated in a realistic way, which

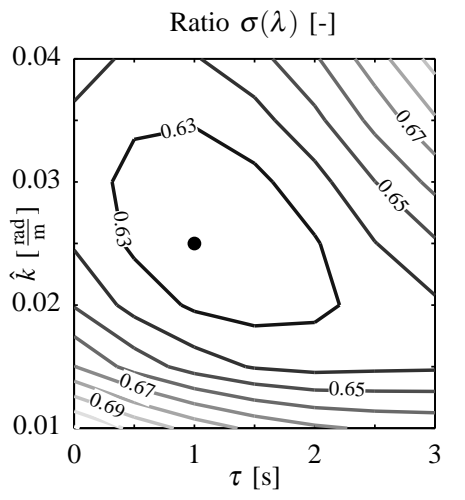

Ratio $\operatorname{DEL}\left(M_{L S S}\right)[-]$

Fig. 9. Relative changes of the DSC in the standard deviation of $\lambda$ and low-speed shaft loads compared to the ISC. Dots: optimal case.

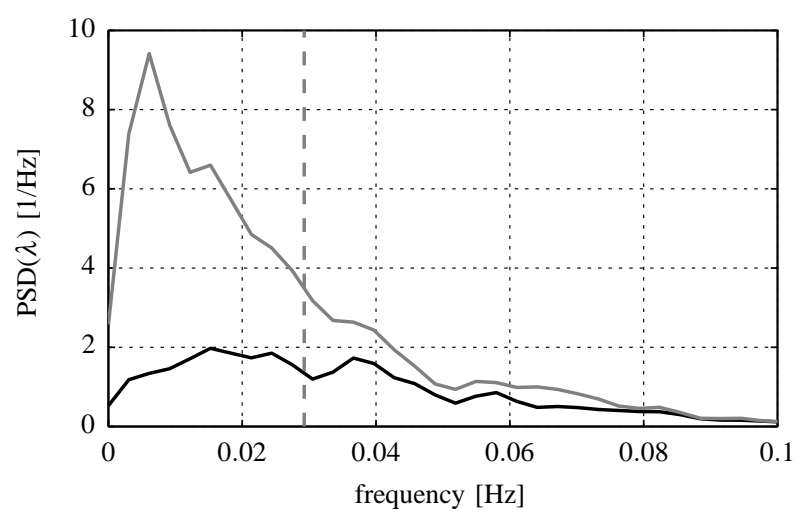

Fig. 10. PSD of the tip speed ratio from Figure 11, ISC (gray) and DSC (black). Average cut-off frequency of the adpative filter (dashed).

effect the DSC would have produced in this specific situation. Furthermore, the DSC can be tuned to the real data.

For better evaluation, the DSC is activated $30 \mathrm{~s}$ after the start and deactivated $30 \mathrm{~s}$ before the end of the simulation. Thus, the differences in energy production is not due to differences in the stored kinetic energy.

Forty-nine simulations with different $\hat{k}$ and $\tau$ are done. Figure 9 shows the changes in the standard deviation of $\lambda$ and damage equivalent loads (DEL) on the low-speed shaft, calculated with Wöhler exponents of 4, typical for steel. The optimal values for $\hat{k}=0.025 \mathrm{rad} / \mathrm{m}$ and $\tau=1 \mathrm{~s}$ from this brute force optimization (minimizing $\sigma(\lambda)$ ) are close to the value from Section IV. Although the $\sigma(\lambda)$ distribution is relatively flat, it is not possible to lower $\hat{k}$ more than approximately $\hat{k}=0.01 \mathrm{rad} / \mathrm{m}$ to reduce the low-speed shaft loads because the resulting time delay of the filter will cause the feedforward signal to be too late.

In Figure 10 and 11 the results of the optimal case can be seen in the time and frequency domain. The fluctuation in $\lambda$ can be reduced by the DSC at the expense of higher fluctuation in the generator torque $M_{g}$. Here, $\sigma(\lambda)$ can be reduced from 0.527 to 0.328 , resulting in a power production increase of $0.3 \%$, which is close to the theoretical value of $0.2 \%$ from Figure 5. Changes may be due to slight changes in the mean value of $\lambda$. 

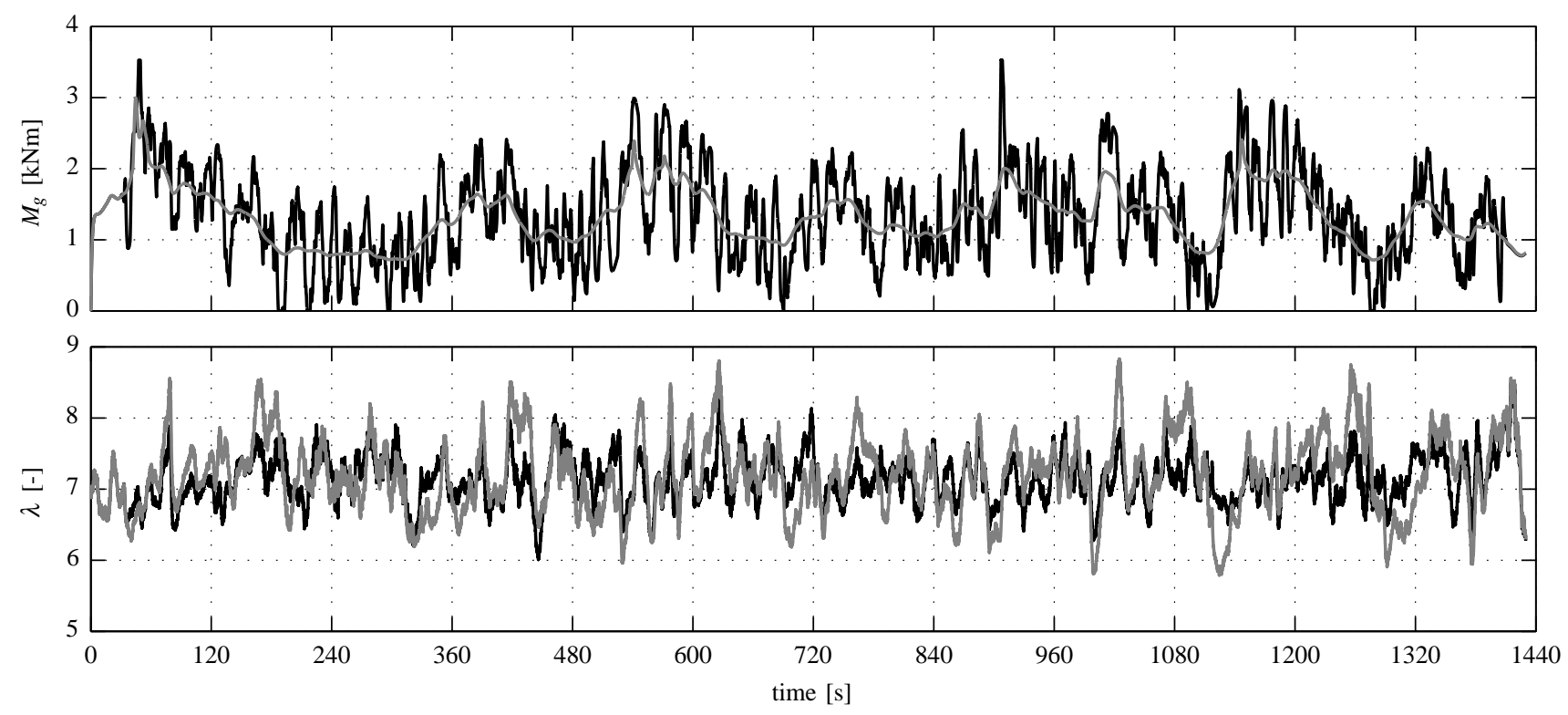

Fig. 11. Simulated reaction of the CART3 to the wind of Figure 6: ISC (gray) and DSC (black).

\section{CONCLUSIONS AND OUTLOOK}

The primary contributions of this paper are the extensions of the previously published direct speed controller. A secondary contribution is the hybrid simulation technique that uses field-test data from a turbine with nacelle-mounted LIDAR in order to carry out a more realistic simulation of LIDAR assisted control.

With sensor data of a research turbine and the simultaneously gathered data from a commercial LIDAR system installed on the nacelle, the controller is optimized to account for the correlation of the LIDAR system and the turbine. The simulations prove that it is important to filter the data according to this specific correlation. The theoretical considerations about the limits of the DSC can be validated. Although a reduction of $37.8 \%$ in the standard deviation of the tip speed ratio can be achieved, the resulting energy gain is only $0.3 \%$. The loads on the shaft are approximately doubled. It was decided that the hybrid simulations sufficiently proved the point that the proposed concept is achievable but not attractive. We believe the results of this study indicate that future research with LIDAR-enhanced control of wind turbines should focus on pitch and yaw control.

\section{ACKNOWLEDGMENT}

Thanks to everyone from NREL, SWE, and Blue Scout Technologies involved in the campaign.

NRELs contributions to this report were funded by the Wind and Water Power Program, Office of Energy Efficiency and Renewable Energy of the U.S. Department of Energy under contract No. DE-AC02-05CH11231. The authors are solely responsible for any omission or errors contained herein.

\section{REFERENCES}

[1] D. Schlipf, P. Fleming, F. Haizmann, A. Scholbrock, M. Hofsäß, A. Wright, and P. W. Cheng, "Field testing of feedforward collective pitch control on the CART2 using a nacelle-based lidar scanner," in Proceedings of The Science of Making Torque from Wind, Oldenburg, Germany, 2012.

[2] A. Scholbrock, P. Fleming, L. Fingersh, A. Wright, D. Schlipf, F. Haizmann, and F. Belen, "Field testing LIDAR based feed-forward controls on the NREL controls advanced research turbine," in 51th AIAA Aerospace Sciences Meeting Including the New Horizons Forum and Aerospace Exposition, Dallas, USA, 2013.

[3] K. A. Kragh, M. H. Hansen, and T. Mikkelsen, "Precision and shortcomings of yaw error estimation using spinner-based light detection and ranging," Wind Energy, 2012.

[4] D. Schlipf, S. Kapp, J. Anger, O. Bischoff, M. Hofsäß, A. Rettenmeier, U. Smolka, and M. Kühn, "Prospects of optimization of energy production by LiDAR assisted control of wind turbines," in Proceedings of the EWEA Annual event, Brussels, Belgium, 2011.

[5] P. Fleming, A. Wright, L. Fingersh, and J.-W. van Wingerden, "Resonant vibrations resulting from the re-engineering of a constant-speed 2-bladed turbine to a variable-speed 3-bladed turbine," in Proceedings of the 49th AIAA Aerospace Sciences Meeting Including the New Horizons Forum and Aerospace Exposition, Orlando, USA, 2011.

[6] T. Burton, N. Jenkins, D. Sharpe, and E. Bossanyi, Wind Energy Handbook. New York, USA: John Wiley \& Sons, 2001.

[7] D. Schlipf, T. Fischer, C. E. Carcangiu, M. Rossetti, and E. Bossanyi, "Load analysis of look-ahead collective pitch control using LiDAR," in Proceedings of the German Wind Energy Conference DEWEK, Bremen, Germany, 2010.

[8] B. Boukhezzar and H. Siguerdidjane, "Nonlinear control of variable speed wind turbines without wind speed measurement," 44th IEEE Conference on Decision and Control and European Control Conference, 2005.

[9] F. Dunne, D. Schlipf, L. Y. Pao, A. D. Wright, B. Jonkman, N. Kelley, and E. Simley, "Comparison of two independent lidar-based pitch control designs," in Proc. 50th AIAA Aerospace Sciences Meeting Including the New Horizons Forum and Aerospace Exposition, 2012.

[10] E. van der Hooft and T. G. van Engelen, "Estimated wind speed feed forward control for wind turbine operation optimization," European Wind Energy Conference, London, vol. 1, p. 9, 2004.

[11] J. Jonkman and M. L. Buhl, "FAST user's guide," NREL, Tech. Rep. NREL/EL-500-38230, August 2005.

[12] E. Bossanyi, "Un-freezing the turbulence: improved wind field modelling for investigating lidar-assisted wind turbine control," in Proceedings of the EWEA Annual event, Copenhagen, Denmark, 2012. 